\title{
Hybrid Tag Anti-collision Algorithms in RFID Systems
}

\author{
Jae-Dong Shin ${ }^{1}$, Sang-Soo Yeo ${ }^{2}$, Tai-Hoon $\mathrm{Kim}^{3}$, and Sung Kwon Kim ${ }^{1}$ \\ ${ }^{1}$ School of Computer Science \& Engineering, Chung-Ang University, Seoul, Korea \\ mulli2@alg.cse.cau.ac.kr, skkim@cau.ac.kr \\ ${ }^{2}$ Department of Computer Science \& Communication Engineering, Kyushu \\ University, Fukuoka, Japan \\ ssyeo@itslab.csce.kyushu-u.ac.jp \\ ${ }^{3}$ Division of Computer Information Communication \& Engineering, Ewha Womans \\ University, Seoul, Korea \\ taihoonn@empal.com
}

\begin{abstract}
RFID, Radio Frequency Identification, technology is a contactless automatic identification technology about which a lot of researches and developments are recently progressing. For this RFID technology to be widely spread, the problem of multiple tag identification, which a reader identifies a multiple number of tags in a very short time, has to be solved. So far, several anti-collision algorithms are developed. And those can be largely divided into ALOHA based algorithm and tree based algorithm. In this paper, two new anti-collision algorithms combining the characteristics of these two categories are presented. And the performances of the two algorithms are simulated.
\end{abstract}

\section{Introduction}

RFID technology is an automatic identification technology of contactless method that identifies electronic tags attached to goods [1]. For this RFID technology to be widely used, multiple tag identification problem must be solved in the first place. This problem is defined as a one-to-many communication problem between a reader and tags. That has to identify by receiving the information transmitted from tags without collision in case a multiple number of tags exist within the identification area of a reader.

Tag anti-collision algorithms can be categorized into ALOHA based algorithms and tree based algorithms. ALOHA based algorithms usually refer to the slotted ALOHA algorithm, an algorithm in which makes only one tag respond in a slot, in the response of tags, by dividing a time into slot units. On the other hand, tree based algorithms make trees while performing the tag identification procedure using a unique ID of each tag.

In this paper, two new algorithms combining the framed slotted ALOHA algorithm, a typical ALOHA based algorithm, and the query tree algorithm, a typical tree based algorithm, are presented. And the performances of the two algorithms are compared through simulations with existing other anti-collision ones used in RFID systems. 


\begin{tabular}{|c|c|c|c|c|c|c|c|c|}
\hline $\begin{array}{c}\text { Forward } \\
\text { link }\end{array}$ & Request & Slot1 & $\begin{array}{c}\text { Ack } \\
1011\end{array}$ & Slot2 & Slot3 & Slot4 & $\begin{array}{c}\text { Ack } \\
0111\end{array}$ & Request \\
\hline $\begin{array}{c}\text { Return } \\
\text { link }\end{array}$ & & 1011 & & Collision & & 0111 & & \\
\hline Tag1 & & & & 0010 & & & & \\
\hline Tag2 & & & & & & 0111 & & \\
\hline Tag3 & 1011 & & & & & & \\
\hline Tag4 & & & 1110 & & & & \\
\hline
\end{tabular}

Fig. 1. An example of tag identification process in FS-ALOHA algorithm

\section{Related Work}

\subsection{Framed Slotted ALOHA Algorithm}

FS-ALOHA algorithm 2] is the most well-known of anti-collision algorithms used for solving the collision of tags in RFID systems. In FS-ALOHA algorithm, when a reader requests tags to transmit their ID, it also transmits a frame size $(F S)$. On receiving ID transmission request from a reader, a tag randomly decides its own transmission slot within the frame size, and then transmits its ID after waiting until its turn. On the reader side, three kinds of cases can occur. To begin with, there is a case receiving no response to the slot. This is referred to as "no response", and the number of no responses in a frame is expressed as $C_{0}$. The second one is a case only one tag has responded. This is referred to as "identification", and the number of identifications in a frame is expressed as $C_{1}$. Lastly, there is a case two tags or more attempt to transmit in the same slot. So, a collision takes place and the data transmitted by tags is lost. This is referred to as "collision", and the number of collisions in a frame is expressed as $C_{k}$.

Fig⿴illustrates the operation of FS-ALOHA algorithm using four tags. The reader requests tags to transmit their ID along with sending 4 as a frame size, and then each tag selects its own slot and attempts to transmit its ID. In Slot1 and Slot4, only one tag attempted to transmit and thus the reader successfully identifies Tag2 and Tag3, then send the tags an Ack command informing it has identified, in order to keep tags from responding in the next frame. In Slot3, however, as there is no tag, it becomes no response. While in Slot2, an collision occurs since Tag1 and Tag4 sent their ID at the same time. Finishing a frame, the reader requests the retransmission of ID to the remaining tags. At this point, the number of remaining tags is estimated using the $C_{0}, C_{1}$, and $C_{k}$, and then the next frame begins by changing to a frame size suitable to the number of remaining tags $\underline{3}$.

\subsection{Query Tree Algorithm}

QT algorithm [5 is a typical one of tree based algorithms. When requesting tags to transmit their ID, a reader sends a prefix $P_{k}$ of $k$ bits together. 


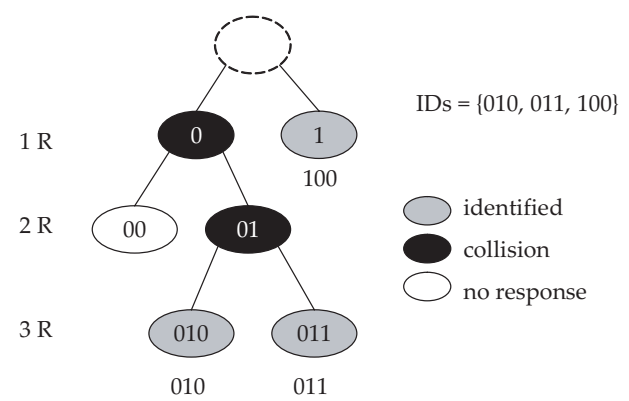

\begin{tabular}{l|c|c|c|c|c|c}
\hline Query & 0 & 1 & 00 & 01 & 010 & 011 \\
\hline Response & collision & identified & no response & collision & identified & identified \\
\hline
\end{tabular}

Fig. 2. An example of identification process in QT [4]

Then each tag confirms whether it is the same as the beginning part of its own ID, and responds its own ID to the reader if it is the same. Likewise as in FS-ALOHA algorithm, at this point, the three cases, "no response", "identification", and "collision", can occur. Here, when a collision takes place, the reader knows there are many tags with the same prefix. In that case, two new prefix $P_{k+1}$ of $k+1$ bits, which "0" and "1" are added at the end of the prefix that has just been transmitted, are made and placed in the queue. The prefix placed in the queue is queried again later. The initial value of the queue is " 0 " and " $1 "$.

Fig2 is an example of having executed QT algorithm on the assumption of three tags whose identifying IDs are "010", "011", and "100", respectively. Prefix "0" and "1" are set up in the initial queue, and the reader queries tags by taking out prefixes in the queue. For a start, when " 0 " is queried, Tags "010" and "011" respond at the same time because the prefix is the same as their ID. Then the reader judges there are two tags or more starting with " 0 " and enters "00" and "01" in the queue. Thereafter, prefix " 1 " is taken out from the queue and queried. Since there is only one tag, "100", it is identified normally to the reader. The first round ends by the method like this. And another round begins for "00" and "01" which has been previously placed in the queue. This algorithm ends when the queue is empty.

\section{Hybrid Anti-collision Algorithms}

In FS-ALOHA and QT algorithm explained above, when there are many tags a reader wants to identify, there exists many tags that will respond at the same time and thus many collisions take place. This ultimately makes the time of identifying tags longer. Framed query tree(FQT) algorithm and query tree ALOHA(QT-ALOHA) algorithm presented in this paper are hybrid forms of FS-ALOHA algorithm and QT algorithm. 


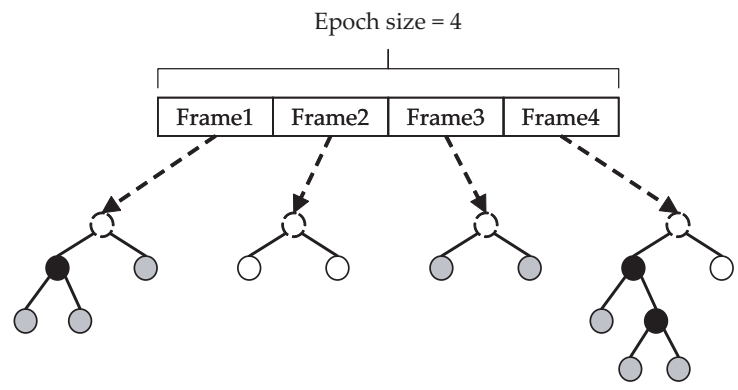

Fig. 3. An example of identification process in FQT algorithm

\subsection{Framed Query Tree Algorithm}

FQT algorithm divides tags randomly into frame units. And within this unit, tags are identified using QT algorithm for them. The actual operation is as follows:

When requesting tags to transmit their ID, a reader also send an epoch size, the number of the total frames. Then each tag decides its own participating frame randomly and responds only when the reader queries its own frame. The reader executes its identification process within each frame using QT algorithm. However, the reader transmit to tags the number of frame as well as the prefix of ID. Each tag confirms whether the number of frame is the same as its own selected number of frame, and if it is the same, transmits its own ID when looking the prefix and it is consistent, as in the existing QT algorithm. After identifying all tags within a frame through QT algorithm identification process in the frame, the reader proceeds to the next frame. This process is carried out repetitively for every frame.

The example of Fig 3 shows the identification process of FQT algorithm when the number of tags to be identified is 8 and the epoch size is 4 . To begin with, a reader transmits the epoch size to tags and then each tag selects its own frame randomly. For three tags which selected Frame1, QT algorithm is used for identifying tags. Thereafter, tags are identified while proceeding to the next frames by the same method.

To determine the most appropriate epoch size is very important in improving performance. By intuition, an epoch size which a tree depth will not exceed 2 when executing QT algorithm exhibits the best performance. The reason is that when executing "0", "1" in the initial queue of QT algorithm, the case that two tags in total, one tag beginning with " 0 " and another tag beginning with "1", are identified is the most ideal. Frame3 is exactly the best case. Assuming the number of tags to be identified is $\mathrm{N}$ and the epoch size is $E S$, the most ideal $E S$ can be expressed as follow:

$$
N=2 * E S
$$


This can be actually verified through the simulation in section 4.1. But there is a big problem with this case. For it is difficult to determine an suitable epoch size from the beginning since the tag identification procedure is initiated under the condition not knowing $N$, in other words, how many tags are to be identified. From that reason, the final FQT algorithm uses FFT(First Frame Test). The FFT begins from a small epoch size and stops its identification process when the first frame has collisions exceeding a collision threshold, and then resumes its identification of tags by increasing the epoch size. As it is assumed that all tags are randomly divided in frames, if many collisions occur in the first frame, remaining frames are more likely to have such a trend.

As shown in the above best case, Frame3 in Fig 3 , a tree can have the best performance when its tags are two with the depth being 1 . Thus a threshold is needed in order to prevent the tree from becoming deeper than this. The collision threshold is a constant based on the concept that the more collisions happen, the deeper the tree becomes. As a result of simulations conducted many times, we have verified that an appropriate epoch size is approached faster than any other cases when this collision threshold is set at 3 . In case it is smaller than 3 , even when the epoch size is adequate, the epoch size becomes big and thus may be passed over. On the contrary, when it is bigger than 3, the epoch size gets too big and thus the speed to increase to an suitable epoch size becomes too slow. So the collision threshold is assumed to be 3 in this paper.

\subsection{Query Tree ALOHA Algorithm}

QT-ALOHA algorithm is another hybrid form of FS-ALOHA and QT algorithm. FQT algorithm basically implements FS-ALOHA algorithm and uses QT algorithm as actual tag identification process. On the other hand, in QT-ALOHA algorithm, QT algorithm is a big picture, while actual tag identification process progresses with FS-ALOHA algorithm. This operation is as follows:

On requesting tags to transmit their ID, a reader sends a prefix and a frame size together. Then only tags which are consistent with their own prefix proceed to FS-ALOHA algorithm with the transmitted frame size. And in the progress of FS-ALOHA algorithm, if a collision takes place even in a single slot, it is interpreted as a collision of QT algorithm, and then a new prefix is made and entered in the queue. At this point, a difference from QT algorithm is to calculate a frame size [3] to be transmitted next and also place this in the queue.

Fig 4 is an example of QT-ALOHA algorithm. It is assumed that the number of tags to be identified is 8 and the initial frame size begins from 4 . In the first round, the reader transmits to tags a prefix "0" and a frame size 4 . And collisions have occurred in the frame. Then, "00" and "01" are entered in the queue and the frame size is determined as 4 through the calculation process of frame size. And then " 1 " is taken out from the queue. When the queue becomes empty, the algorithm ends. 


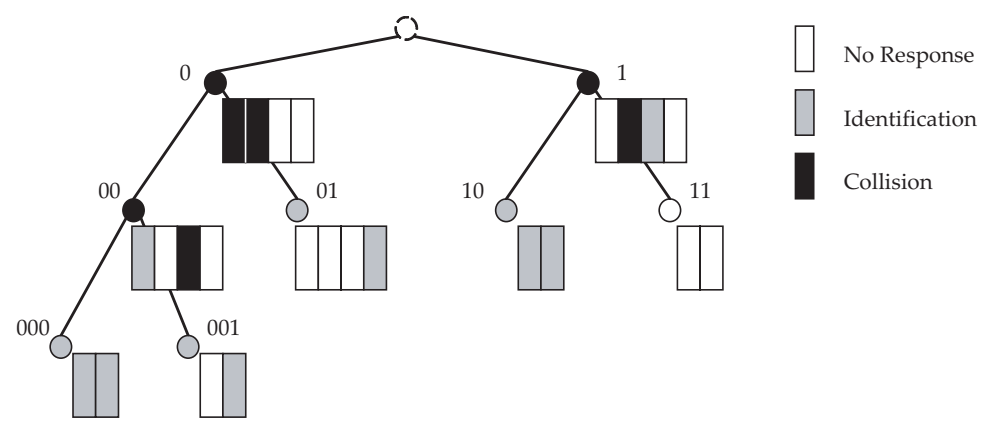

\begin{tabular}{l|c|c|c|c|c|c|c|c}
\hline Query & 0 & 1 & 00 & 01 & 10 & 11 & 000 & 001 \\
\hline Frame Size & 4 & 4 & 4 & 4 & 2 & 2 & 2 & 2 \\
\hline Response & collision & collision & collision & identified & identified & no res. & identified & identified \\
\hline
\end{tabular}

Fig. 4. An example of identification process in QT-ALOHA algorithm

\section{Simulations}

The ID of tags used in simulations was set at a size of 64 bits according to the international standard and was created using random number generator. And for raising the reliability of tag simulation results, 100-time simulations were conducted for every same environment and average it.

\subsection{Epoch Size of FQT Algorithm}

The first simulation was conducted with the epoch size being changing under the condition of 100 tags. Table 1 shows the results of this simulation. Reviewing this results, they can be divided into three cases according to the epoch size. The first case is at 32 and 64, close to about 50, an ideal epoch size. This case demonstrates the best performance as we mentioned in section 3.1. The second is a case smaller than the ideal epoch size. But in this case, an optimum epoch size is found by the first frame test. And the last is a case bigger than the ideal epoch size. As there is no operation to reduce the epoch size in FQT algorithm and thus the initial epoch have to be entirely executed.

Table 1. Performance comparison according to Epoch Size(ES)

\begin{tabular}{lllll}
\hline Frame Size & Query & $C_{0}$ & $C_{1}$ & $C_{k}$ \\
\hline 16 & 246.6 & 55.1 & 100.0 & 81.2 \\
\hline 32 & 238.2 & 56.9 & 100.0 & 78.2 \\
\hline 64 & 241.3 & 85.9 & 100.0 & 54.1 \\
\hline 128 & 327.2 & 193.6 & 100.0 & 32.4 \\
\hline 256 & 550.2 & 430.6 & 100.0 & 18.6 \\
\hline
\end{tabular}




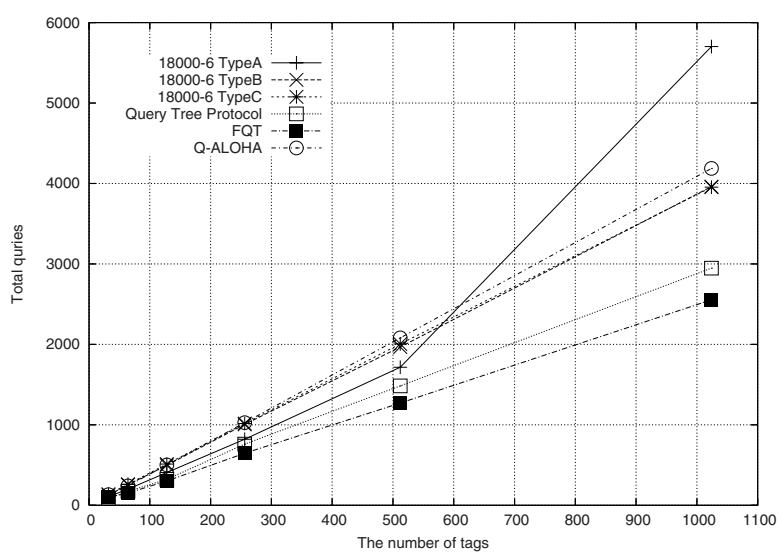

Fig. 5. Comparison of query-response number

\subsection{Performance Comparison Between Presented Algorithms and Other Ones}

The second simulation has compared the presented FQT algorithm and QTALOHA algorithm with other anti-collision algorithms, 18000-6 [6] Type A, Type B, Type C, and QT algorithm. This comparison sets the query-response number of times between a reader and tags as a comparison value while changing the number of tags from 32 to 1,024. On the other hand, in the algorithms based on FS-ALOHA algorithm, such as Type A, Type C and QT-ALOHA algorithm, the initial frame size is started arbitrarily at 32 regardless of the number of tags. The epoch size in FQT algorithm also begins at 32 .

Fig 5 and 6 are the results of simulations. 18000-6 Type A and Type C use the same operating method, FS-ALOHA algorithm. But as the maximum frame size of Type A is 256, if executing the algorithm at the number of tags higher than it, a significant degradation in performance can be seen. Type $\mathrm{C}$, however, if there occurs many collisions or no-responses even in the middle of a frame, stops the on-going frame and proceeds to the next frame, thereby improving the performance. Type B uses binary tree algorithm, one of the tree based algorithms.

The results show that the tree based algorithm typically is lower in the queryresponse number of times than the ALOHA based algorithms. For tags identified in a frame of the ALOHA based algorithm send tags a command they have been identified so they cannot be included in the identification process of the next frame.

Fig 6 illustrates how many queries-responses are needed in order to identify one tag. Looking into this, it can be seen that FQT algorithm queries less for identifying one tag than any other algorithms. Its performance improvement is 10 to 50 percent of times than many other existing anti-collision algorithms. 


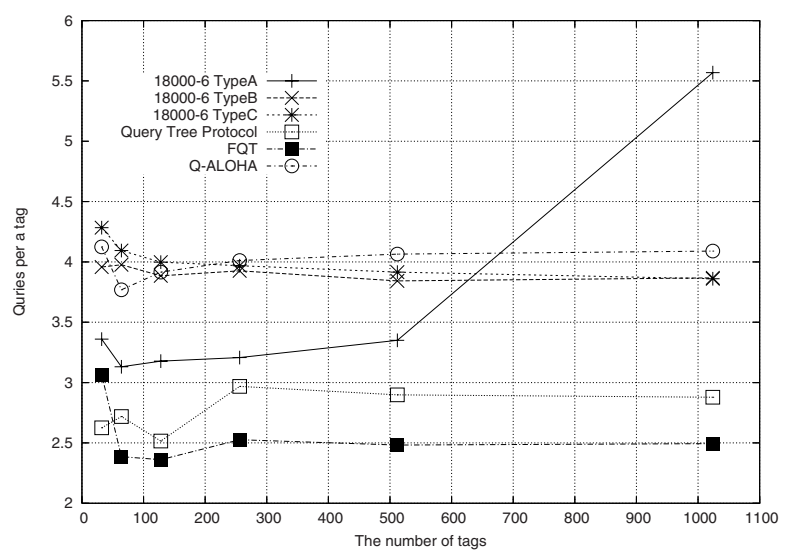

Fig. 6. Comparison of query-response number needed for identification per tag

\section{Conclusions}

Two new algorithms combining the characteristics of the two categories, ALOHA based and tree based algorithm, have been presented in this paper. FQT algorithm of the two, in particular, has shown a big performance improvement. In the near future, methods to find a faster optimum epoch size in FQT algorithm are to be studied.

\section{Acknowledgement}

This work was supported by grant No. R01-2005-000-10568-0 from the Basic Research Program of the Korea Science \& Engineering Foundation.

\section{References}

1. K. Finkenzeller, "RFID handbook", John Wiley \& Sons, 1999.

2. F. C. Schoute, "Control of ALOHA Signalling in a Mobile Radio Trunking System", International Conference on Radio Spectrum Conservation Techniques, IEEE, pp.38-42, 1980.

3. H. Vogt, "Multiple object identification with passive RFID tags", In IEEE International Conference on Systems, Man and Cybernetics (SMC'02),October 2002.

4. J. Myung and W. Lee, "An Adaptive Memoryless Tag Anti-Collision Protocol for RFID Networks", IEEE 24th Conference on Computer Communications (INFOCOM'05), March 2005.

5. C. Law, K. Lee, and K. Siu, "Efficient Memoryless Protocol for Tag Identification", 4th International Workshop on Discrete Algorithms and Methods for Mobile Computing and Communications, pp.75-84, ACM, August 2000.

6. ISO/IEC 18000-6:2004/Amd 1:2006, International Organization for Standardization, June 2006. 